\title{
Optimization of Energy Efficient Advance Leach Protocol
}

\author{
1. Sandeep Kaur, 2. Dr. Rajeev Bedi, 3. Mohit Marwaha
}

Abstract: In WSNs, the only source to save life for the node is the battery consumption. During communication with other area nodes or sensing activities consumes a lot of power energy in processing the data and transmitting the collected/selected data to the sink. In wireless sensor networks, energy conservation is directly to the network lifetime and energy plays an important role in the cluster head selection. A new threshold has been formulated for cluster head selection, which is based on remaining energy of the sensor node and the distance from the base station. Proposed approach selects the cluster head nearer to base station having maximum remaining energy than any other sensor node in multi-hop communication. The multi hop approach minimizing the inter cluster communication without effecting the data reliability.

Keywords: Optimization, Energy Efficient, Advance Leach Protocol, WSN, Multi-hop Communication, Wireless Sensor Network

\subsection{INTRODUCTION}

\subsubsection{Wireless Sensor Networks}

Wireless Sensor Networks (WSNs) comprise of tiny, batteryoperated sensor nodes (SNs) with limited process involvement, storage \& radio abilities. WSNs may contains hundreds or thousands of multi-functional power SNs which works in harsh environments. They have the capability to sense and perform computations. SNs sense the data and transmit the reports belonging to the sensed data to sink node. The SN components includes: a Central Processing Unit (CPU), a sensor device unit, a power unit for energy, a communicating unit and an analog to digital (A to D) converter. The miniatured SNs embedded are micro-electromechanical systems (MEMS) which are actually accountable to make a calculable alteration in some physical situations linked with pressure \& temperature. The SNs computes by sensing the physical data of the monitored part. The tiny SNs senses the analog signals and are then digitized by A to D converter and transmits to a controller for execution. The SNs of small size are low powered and are operated in high volumetric density. These SNs can be independent of an adaptive surrounding and its customary architecture utilizes costly macro-sensors to receive accurate information. For illustration, the gas \& oil companies employed large number of sensors (geophone) linked by cables/wires to perform oil investigation. The SNs needs huge energy or power for their operation. One of the limitations is to encounter controlled operation in WSNs. Owing to the high utilization of WSNs, it also faces the problem of control energy restraints particularly in battery limitations. As the SNs are have lot of major issues in WSNs, the failure of one node due to energy can interrupt the complete working environment. In Inactive mode, the SNs are in a processing state in which energy consumption during receiving or transmitting of data takes place. In sleep mode, the SNs are shut down for saving an energy. Constraints related to power end by generating further computational and storage limitations which leads to architectural problems. Platform of WSNs should offer support for applications of specific protocols which minimize node's size, cost, and power consumption.

The steps given below assists in saving energy consumed by communication in WSNs:

- Modifying the transmission range within the SNs.

- To change the state (transmitting, receiving, idle or sleep) of SNs.

- Proper data collection schemes with utilization of appropriate routing algorithm.

- Circumventing the management of meaningless data. In WSNs, the only way to save battery of SNs is to diminish the consumption of battery. As communication within SNs or sensing activities uses immense power during the data processing and transmission, the battery re-charging or saving becomes utmost important. In defence application and surveillance applications it is hard to alter the batteries that 
drains out [4]. Myriad of scientists are thus finding out energy-aware ways for WSNs to mitigate this limitation of energy efficiency.

Some soft and real-time support must be provided by the protocols designed in these areas in WSNs. The applications in these areas performs the task of data processing, sensing and transmitting the data. The base station (BS) or sink employs the data collected by all the detecting SNs to organize in proper way.

\subsubsection{Wireless Sensor Networks VS Wireless AD-Hoc}

\section{Network}

Remote Sensor Systems have been considered as one of the foremost critical exceptionally later advances for the twenty - to begin with century. Empowered by later development innovations in microelectronic mechanical frameworks and remote communication innovations, tiny/small, cheap/low taken a toll, and savvy information sensors conveyed in a physical range arrange and organized through a remote joins and the based Web give uncommon openings for a assortment of people/civilian and discuss protection applications, for illustration, natural observing, resistance fight field observation, AI mechanical autonomy and mechanical handle control framework. A gigantic sum of analyst exercises have been carried out by, to clarify, to investigate and to fathom different arrange plan issues and application issues, and critical progresses have been made within the advancement arrange and arrangement of sensors in WSNs. Close to of this conventional remote communication systems, for case, neighbourhood cellular frameworks and portable ad hoc network (MANET), WSNs have a special characteristic, for case, denser organize level and number of information node sending, higher instability of information sensor nodes, and serious control vitality framework, computation, and tremendous capacity imperatives, which display many new level challenges within the advancement of arrange and application of WSNs.

\subsubsection{Design Goals for WSN}

The main section of network is the clustering protocols which are one of the routing protocols of WSNs. There are various factors that affect the working of routing protocols in WSNs, so, before moving further, the detailed study of about such factors are reviewed. The performance efficiency of WSNs depends upon the following factors:

\section{a) Use of network power}

Network energy is used by all the SNs deployed in the defined area that assists these nodes to perform functions like: sensing, data processing or formation of groups in a network.
The network power use is defined as the total amount of energy used by the SN within that network.

\section{b) Energy Awareness}

For data sensing, its processing, storage and transmission, every node in a network area makes use of power. A SN in a network area must know the amount of energy that once utilized by $\mathrm{SN}$ in order to perform a new task. The type of functionality determines the amount of power energy that is distributed i.e it could vary from high to low or moderate.

\section{c) Transmission Method}

SNs that gathers the transmitted data and sends it to the BS by making use of multi-hop routing method or flat routing.

\section{d) Easy Installation}

SNs consists of thousands of SNs that may require the node to be deployed in the remote areas/fields. Hundreds to thousands of SNs can be easily thrown from a plane over a remote field where human reach is difficult. It permits us to gather information that otherwise would be impossible.

\section{e) Synchronization}

When SNs guarantee that receiver side could identify the data transmitted in an exact order like it was sent. This process is called synchronization i.e when the sending and receiving flow rate is almost similar between the two SNs in a network. The SNs in this case ought to have similar time notion that is sleep and wake up state.

\section{f) System lifespan}

The WSNs must operate for longer duration and the total lifetime of system in a network area must be computed by making use of parameters like: time for node to become dead. It can also be computed using parameters such as: time until the $\mathrm{SN}$ gives unacceptable results.

\section{g) Processing time of node}

The entire time taken by the SN in WSN for accomplishing all the functions initiating from its first activity of sensing to its processing or storage within the buffers is known as processing time of node.

\section{h) Latency}

Data packets from SNs are classically time-sensitive, therefore it is quite significant to take a data packet in an appropriate manner i.e no time delay should be there. Long latency because of processing or communication is undesirable. 


\section{i) Quality}

There are some quality parameters that must be matched with the data that has been sensed by the SNs in order to prove the accuracy. The various applications of WSNs have distinct Quality of Service (QoS) needs such as: loss of data packet and delay time. One of the examples of this is: fire monitoring in which timely delivery of service is important and it is very sensitive application. Therefore, QoS parameter is one of the imperative requirements of the designing of WSNs.

\section{j) Latency}

The time taken by the $\mathrm{SN}$ to sense the data is known as Latency. It totally relies upon the type of application being used. SNs gathers the data and subsequently process it and transmit the same to the BS. It is computed based on the activities and the time a SN takes to send the data packet in loaded environment or where there is a low traffic.

\section{k) Channel Utilisation}

SNs are quipped with limited bandwidth resources. Therefore, an efficiency must be considered while designing the communication protocols for WSNs and this can be possible if proper bandwidth is used with an objective of improving channel utilization.

\section{l) Self Configurability}

Mostly, the SNs are deployed without planning or engineering in WSNs. But it is mandatory to carefully plan the design and deployment positions for efficient results because once the deployment of SNs is done, it should be capable of organizing themselves without failure or must reconfigure itself if any topological changes occur.

\section{m) Small Node Size}

One of the main design aims of WSNs is to minimize the size of SN, so that large number of SNs could be deployed easily in a hostile environment. Minimizing the size of SN can considerably reduce its associated deployment cost as well as low battery consumption takes place.

\section{n) Low Node Cost}

SNs cost reduction is another chief concern of WSNs. As the SNs are placed in those regions where the environment is harsh, so the nodes deployed once cannot be reused. Thus, it is very important to minimize the cost of $\mathrm{SN}$ in order to reduce the cost of entire WSNs, so that large number of nodes can be deployed.

\section{o) Fault Tolerance}

Owing to the dangerous or rough environment, the SNs are sensitive to the failure and therefore it becomes important to deploy those SNs which are robust and less prone to failure. It becomes necessary to use SNs that have the abilities of selfimprovement in terms of repair, recovering and testing.

\section{p) Security}

There are high chances of security breaches for the SNs that are deployed for military applications. Therefore, it is vital to introduce effective security features to prevent an unauthorized information leak. The implementation of high security mechanisms would upbring the security level and would make the network more secure and robust.

\section{q) Scalability}

In WSNs, scalability is an imperative factor as the it is not obligatory that the network is static. There might be several situations where the network is dynamic and it changes as per the requirements given by the user. Thus, it is necessary that the SNs must have scalable nature such that it can adjust to the changes or requirements in the network.

\section{r) Low Power Consumptions}

One of the vital aims of the WSNs is to design a network that consumes lesser power. Moreover, the SNs are battery operated and they require frequent change of their batteries which is actually very difficult task. Thus, reducing the amount of energy or charging SN from distant are the only two ways to extend the lifespan of WSNs.

\section{r) Scalability}

The large number of SNs are deployed in a network ranging from hundreds to thousands. Thus, a scalable design of network is mandatory to adjust the protocol in accordance with the varying network sizes.

\section{s) Adaptability}

It is very crucial for the SNs to adapt to the changing environments in a network, for example topological changes might disturb the network, therefore the SNs must be such that they can adapt to the changes. Thus, adaptive nature of SNs in terms of density and topological changes could improve the lifespan of WSNs

\section{t) Reliability}

There could be myriad of situations which could affect the reliability of WSNs such as: noise, error and delays. To overcome such issues, the protocols in WSNs must provide an error correction method to guarantee reliable data transmission service. 


\subsection{Problem Formulation}

WSNs area involve thousands to millions of sensor data nodes, with a restricted energy. As there are different clustering protocol in wireless sensor area networks used for cluster area head selection, some depends it upon the average energy of a node and some protocols depends upon probability basis. These protocols works either on homogeneous or heterogeneous networks, For Paper "Energy Efficient Data Communication Approach in WSN" In this paper the node Id is missing on the basis of location (have not consider) so, this portion of energy loss will be conserved (which will loss in framing the data packet into the payload and at the time of data aggregation).

This approach is failed when a malicious node is in the sensor network which continuously send the hello packet to the networks (flooding attack), because on the basis of Id we can detect the malicious node in a routing path. System Model is similar to W. B. Heinzelman, A. P. Chandrakasan, H. Balakrishnan, "An application specific protocol architecture for Wireless Microsensor Networks"

\subsection{Objective}

The following are the objectives of my work

1) To study the various energy efficient routing protocols in WSN.

2) To design the energy efficient clustering strategy for the deployment of sensor nodes.

3) To simulate the proposed approach and evaluate its performance and to compare the proposed technique with the conventional technique.

By doing these objectives it will improve the overall WSN life time and energy consumption with sensor node.

\subsection{Proposed Algorithm}

Below are some mandatory steps that could be followed in order to save energy in WSNs:

1) Scheduling the among the state of transmitting, receiving, idle and sleep.

2) Modifying the transmission range amid the SNs.

3) Making use of effective routing strategy with improved method of data collection.

4) In case of overhearing, the meaningless/unwanted data must be avoided.

Battery utilization in effective way is the only remedy to save SNs from dying in WSNs. When communication takes place within the SNs, ample of power is consumed in the phase of data processing and transmission or reception to the BS. However, it is quite challenging to replace the batteries of such sensing nodes which die off from extra power utilization. Saving the battery is thus very crucial part specially for critical applications which need immediate attention such as: defence, surveillance applications and many more. It is hard to substitute the batteries gets drained off and therefore various researchers are continuously working on to improve the power through energy-aware protocols in WSNs so as to outweigh the energy issues appropriately.

Some real-time supports are being provided in all the protocols designed for WSNs as there are certain applications in these areas where SNs sense, processed and transmit data to other SNs within a network. Therefore, quick and reliable reactions are indeed important in protocols which are said to provide real-time assistance in improving the network lifespan. A redundant and identical data must be sent to the BS. It should provide a redundant and duplicate data to the BS. The BS makes use of the data being collected by the SNs in a network. The transmission delay of analysed data to the BS from the SNs must be small to generate quick reply.

\section{Working flow:}

Deployment $\rightarrow$ Sensing $\rightarrow$ Network Setup $\rightarrow$ Root $\rightarrow$ Data Aggregate $\rightarrow$ Cluster Head Selection $\rightarrow$ Routing Path Selection $\rightarrow$ Downlink $\rightarrow$ Data Aggregation $\rightarrow$ Cluster Head Selection $\rightarrow$ Routing Path Selection $\rightarrow$ Uplink then whole the process will repeat again and again until all the nodes are dead.

Energy Model

Based on LOS or Two ray propagation model.

$$
\begin{aligned}
& E_{t x}(i, j)=\left(\alpha_{t x}+\epsilon_{f s} D_{i j}^{2}\right) * \mathcal{L}, \\
& \left(\alpha_{\mathrm{tx}}+\epsilon_{\mathrm{mp}} \mathrm{D}_{\mathrm{ij}}{ }^{4}\right) * \mathcal{L}, \\
& \mathrm{E}_{\mathrm{rx}}=\alpha_{\mathrm{rx}} * \mathcal{L} \\
& \mathrm{D}_{\mathrm{ij}}<\mathrm{d}_{0} \\
& D_{i j} \geq d_{0}
\end{aligned}
$$

Deployment of Node

May be solid angle based (Proposed) for adaptive sensing

Random basis or

Grid Basis

Clustering Algorithm (proposed)

$\mathrm{T}(\mathrm{i})=\rho(\mathrm{i}) * \mathrm{~T}_{\mathrm{cH}}$

Or

$\mathrm{T}(\mathrm{i})=\frac{\sum_{\mathrm{i=1}}^{\mathrm{N}} \mathrm{m}_{\mathrm{i}} * \mathrm{r}_{\mathrm{i}}}{\mathrm{m}_{\mathrm{i}}}$

Or

Power radiated by B.S to the node (Antenna lobe beamforming)

Which is convenient?

Time complexity

May be TDMA scheduling

Or

New proposed 


\section{Data Aggregation}

Existing or proposed

Routing Protocol (Down-Link and Up-Link)

Existing or proposed

Or

\section{TDMA || FDMA || CDMA || SDMA}

Which balanced the energy?

Comparing results with the base paper or existing protocols.

\subsection{Programming Environment}

MATLAB will be utilized as test bed to execute the required objective proposed within the paper. MATLAB may be a high-level dialect and intuitively environment that empowers you to perform computationally seriously assignments which can be speedier than with programming dialects such as $\mathrm{C}++$, C, and FORTRAN. MATLAB stands for "Lattice Research facility" and may be a most numerical computing environment and unused fourth-generation programming dialect. Typically created by The Math Works, it permits lattice controls, like plotting of charts, capacities and information, usage of strategy calculations, creation of modern client interfacing, and meddle with programs composed in other dialects, like $\mathrm{C}, \mathrm{C}++$, and FORTRAN.

In spite of the fact that the basically expecting of MATLAB is to computing numerical values, an discretionary tool compartment in MATLab employments the MuPAD typical motor, which permits get to to typical computing capabilities. Along this an extra bundle, like Simulink, includes graphical multi-domain recreation and other like Model-Based Plan for energetic and implanted frameworks. In 2004, Math Works claimed that MATLAB was being utilized by more than one million individuals over the world in industry and the scholastic areas. MATLAB clients come from different foundations of building, science and financial matters.

MATLAB is an ambitious program. It contains hundreds of commands to do mathematics. We can use it to graph functions, solve equations, perform statistical tests and do much more. It is a high level programming language that can communicate with its cousins e.g., FORTRAN and C. We can also produce sound and animate graphics. We can do simulations and modeling (especially if we have access to not just basic MATLAB, but also to its accessory SIMULINK). We can use MATLAB in conjunction with the word processing and desktop publishing features of Microsoft Word, to combine mathematical computations with text and graphics to produce a polished, integrated and interactive document. It is very easy to install this software on your computer. It can be installed by running the set up file.

\subsection{Proposed work Implementation and Results}

In this section the comparison of proposed technique with LEACH, and SEP is discussed. The comparison will show the improvement on network life time and throughput of network affected with the help of residual energy and compression.

The evaluation and the implementation is done in MATLAB. The simulation has been peformed in the network of 100 nodes and are placed randomly in the network. The nodes are in the diameter of field $400 \mathrm{~m} \times 400 \mathrm{~m}$.

The different value of parameters used in the network is shown Table 5.1. the comparison of different metrics of the protocols are dead node evaluation, alive node evaluation, packets to $\mathrm{CH}$, packets to BS and number of cluster heads.

Table 5.1 Value of Parameters used

\begin{tabular}{|l|l|}
\hline Parameter & Values \\
\hline Area (x, y) & 400,400 \\
\hline Base station (x, y) & 200,200 or mobile \\
\hline Nodes (n) & 100 \\
\hline Probability (p) & 0.1 \\
\hline Initial Energy & $0.5 \mathrm{~J}$ \\
\hline Transmitter energy & $50^{*} 10^{-9}$ \\
\hline Receiver energy & $50^{*} 10^{-9}$ \\
\hline Free space(amplifier) & $10^{*} 10^{-12}$ \\
\hline Multipath(amplifier) & $0.0013^{*} 10^{-12}$ \\
\hline Effective Data aggregation & $5^{*} 10^{-9}$ \\
\hline Maximum lifetime & 2000 \\
\hline
\end{tabular}

The network consists of 100 nodes and the deployment of the of nodes having cluster heads and normal nodes are shown in figure 5.1.

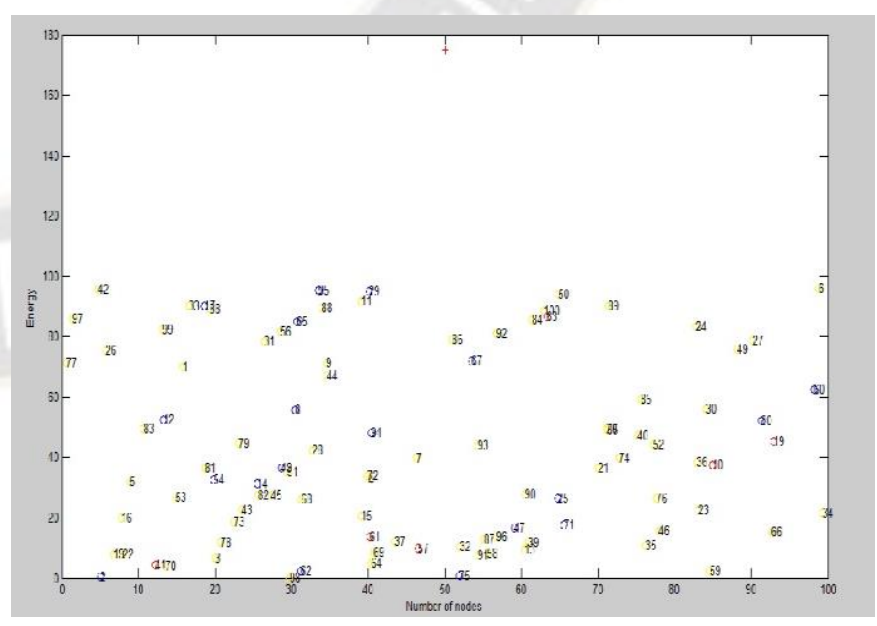

Figure 5.1 Deployment of sensor nodes 
Data sending from cluster heads to cluster heads are shown in figure 5.2 shown by links and data transmission from cluster head to neighbouring nodes are shown in figure 5.3.

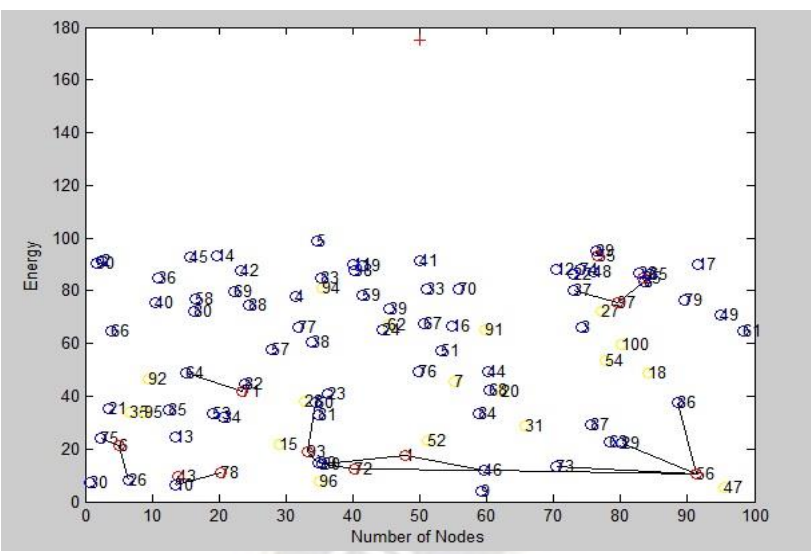

Figure 5.2 Data sending CH1 to CH2

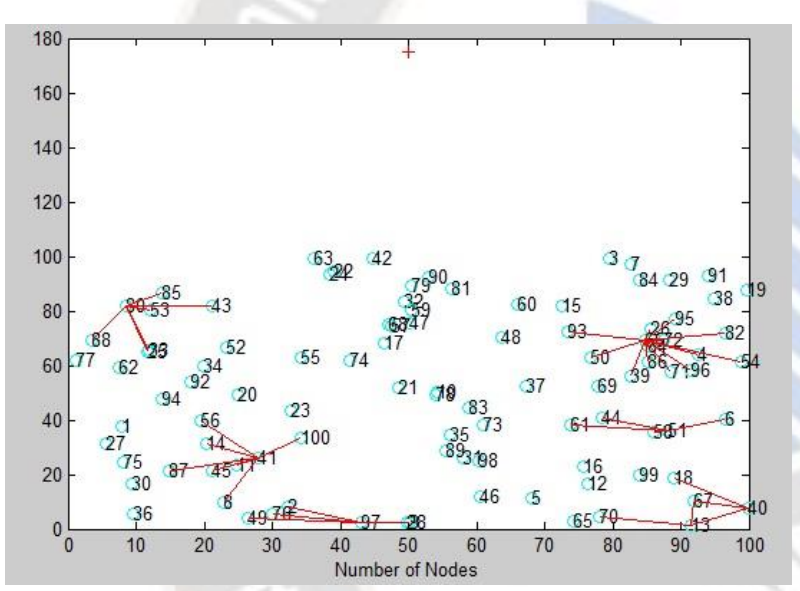

Figure 5.3 Data sending CH to neighbour nodes

The complete data sending from $\mathrm{CH}$ to nodes are shown in figure 5.4.

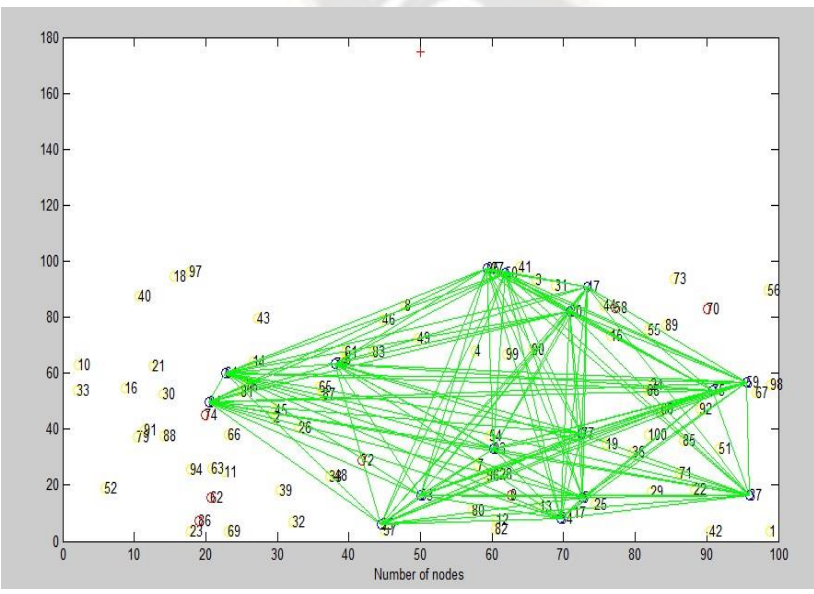

Figure 5.4 Data sending to $\mathrm{CH}$
Data transmission from $\mathrm{CH}$ to base station is shown in figure 5.5 and figure 5.6.

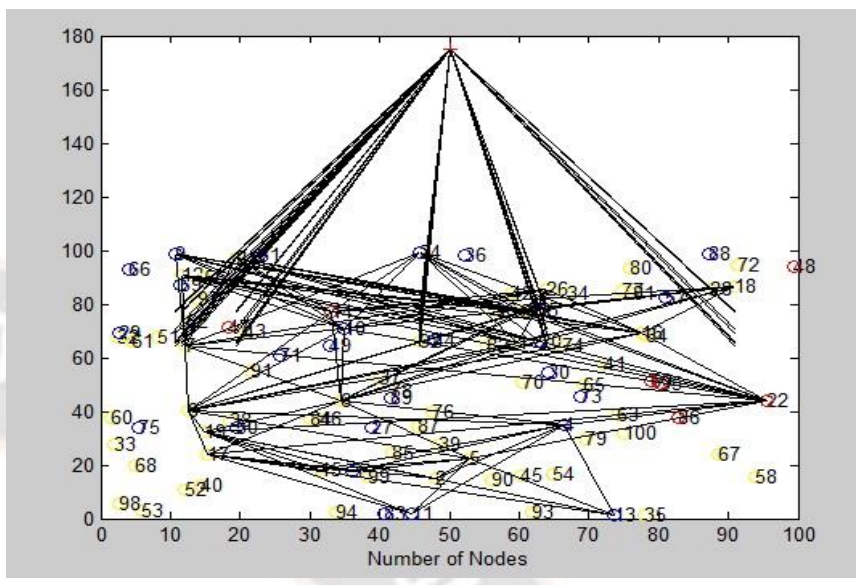

Figure 5.5 Data sending to BS

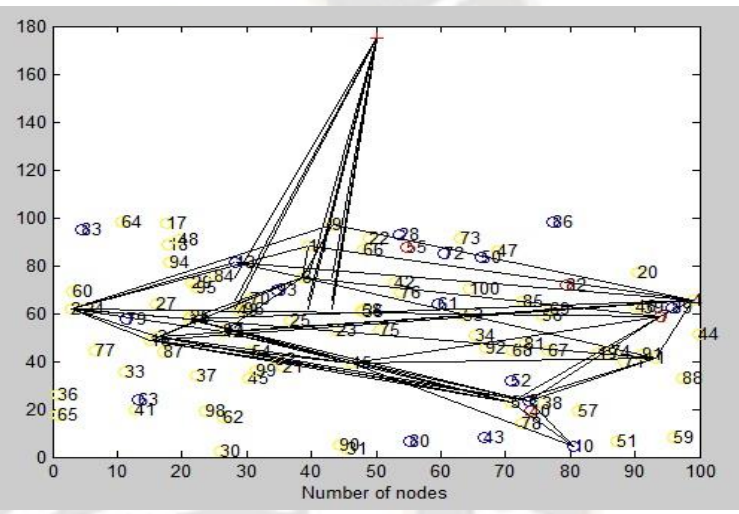

Figure 5.6 Data sending in rounds to BS

The stability period and network lifetime are used as key indicators to estimate performance of the proposed approach. The stability period shows that the time interval from the start of the operation to the first node dies. In 2000 rounds the stability of the network and set of alive nodes are shown in figure 5.7 and figure 5.8 .

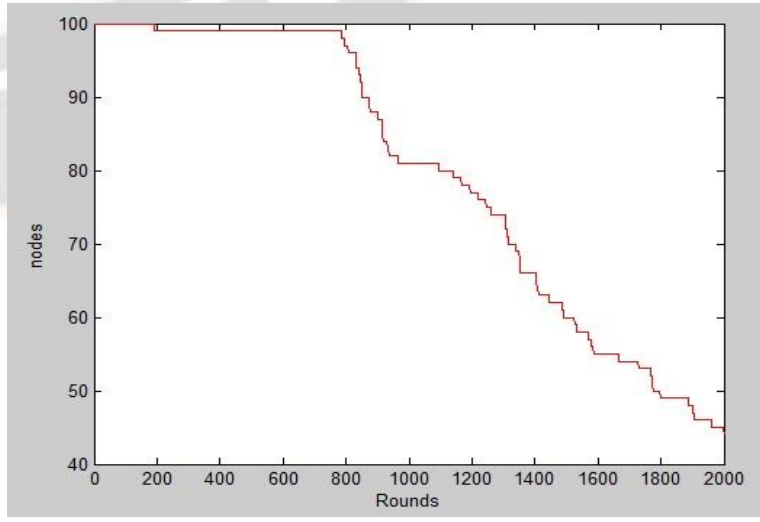

Figure 5.7 Stability of nodes 


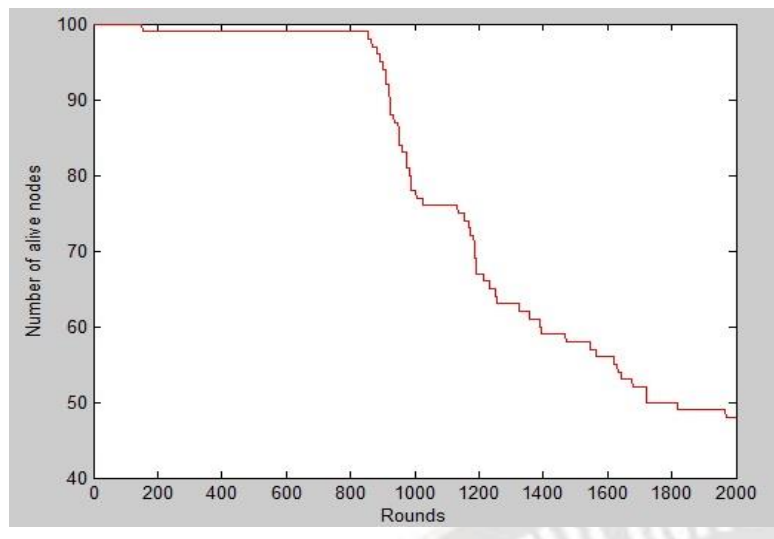

Figure 5.8 Alive nodes

The concept of multi-hop using on previous deployment technique show the improvement over the network life time and enhances the chance of efficiency of the network as shown in figure 5.9, figure 5.10, figure 5.11, figure 5.12, figure 5.13 and figure 5.14 .

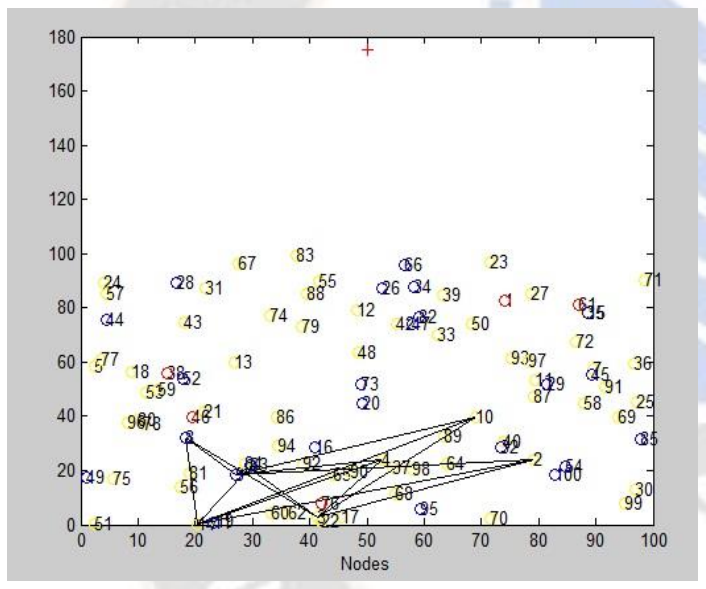

Figure 5.9 Multi hop level1

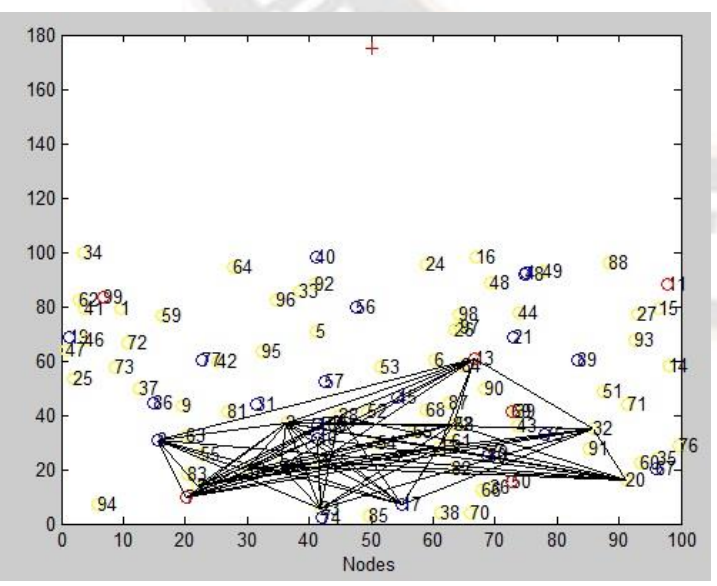

Figure 5.10 Multi hop level2

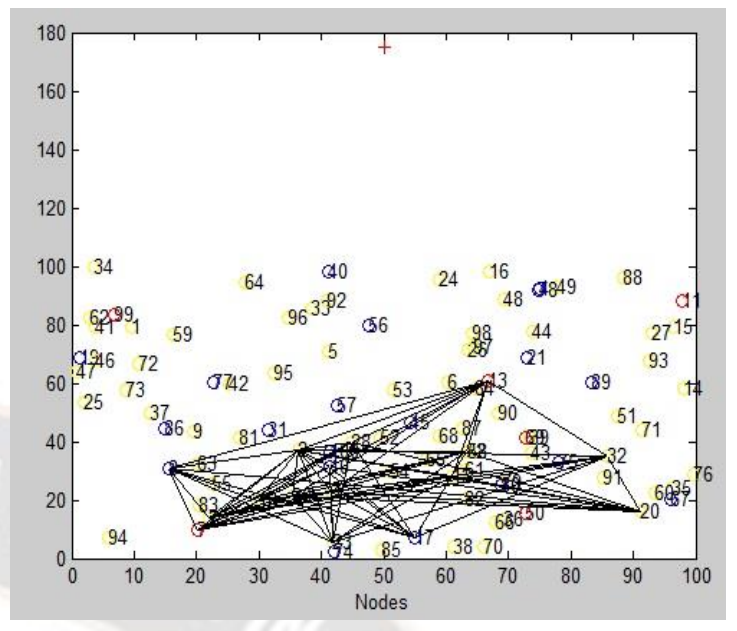

Figure 5.11 Multi hop level3

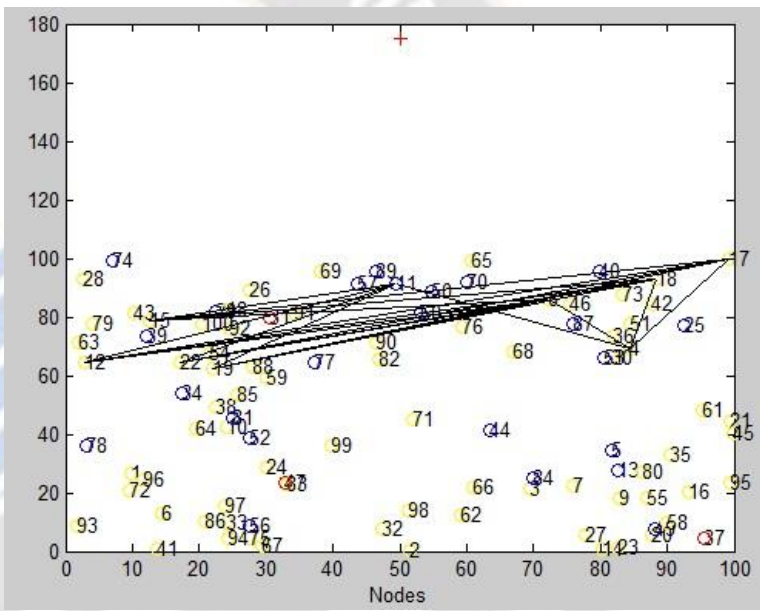

Figure 5.12 Multi hop level 4

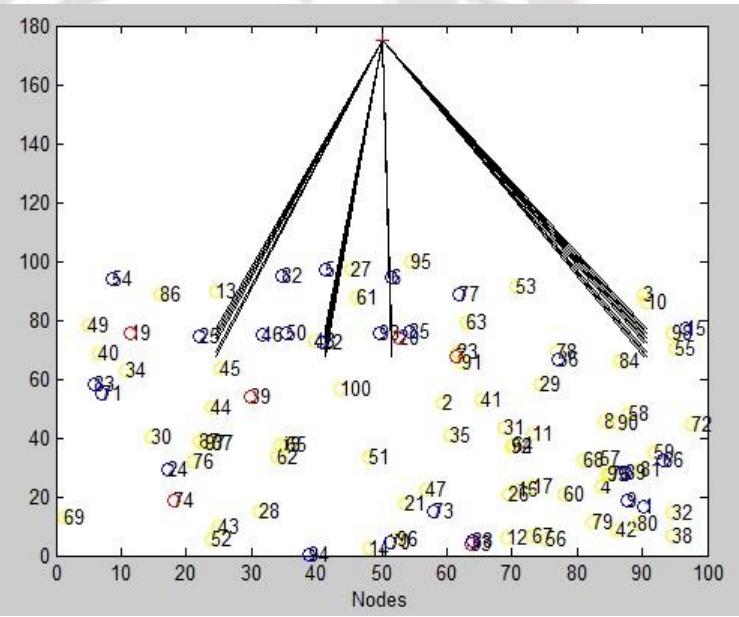

Figure 5.13 Multi hop level 5 


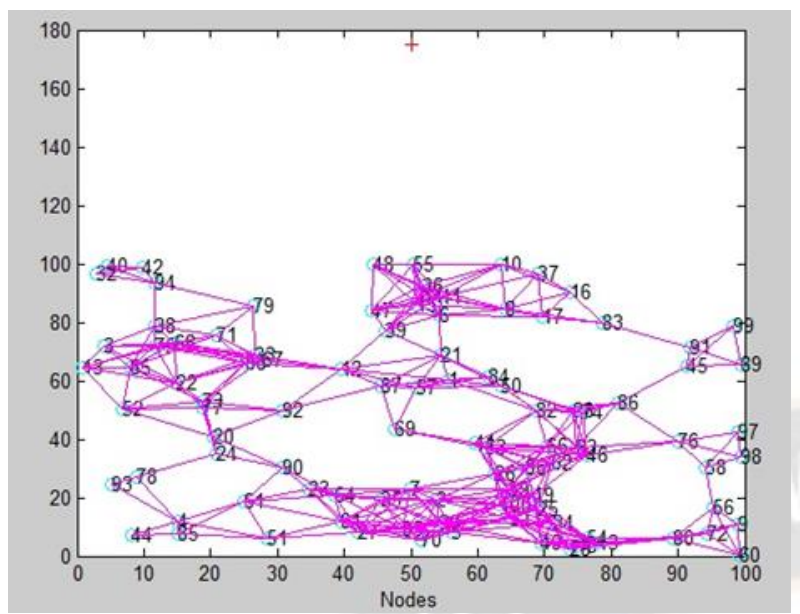

Figure 5.14 Multi hop deployment

\section{Comparative Analysis of Node Energy Efficiency show} the following result

\begin{tabular}{|l|l|l|}
\hline Comparison(rounds) & Leach & $\begin{array}{l}\text { Proposed } \\
\text { Protocol }\end{array}$ \\
\hline First Node Died & 948 & 1383 \\
\hline Last node Died & 2593 & 3412 \\
\hline $\begin{array}{l}\text { Average Life of The } \\
\text { node }\end{array}$ & 2184 & 2732 \\
\hline
\end{tabular}

\subsection{Conclusion}

In WSNs, the only source for saving life for the SNs is the optimum utilization of battery. During communication with SNs, sensing process use lot of battery power and therefore sends the gathered data to the BS. In wireless sensor networks energy conservation is directly related to the network lifetime and also energy play an important role in the cluster head selection. A new threshold has been formulated for cluster head selection which is based on the remaining energy of the sensor node and the distance from base station. Proposed approach selects cluster head nearer to base station having maximum remaining energy than any other sensor nodes in multi-hop communication. The multi-hop approach minimizing the inter cluster communication without effecting the data reliability. The main objective is to find ways to distribute the cluster head throughout the sensing field that will improve energy efficiency and help in reliable transmission of sensed data to the base station in wireless sensor networks.

\subsection{Future Work}

In future work the compression technique can be used to maximize the data transmission so that the maximum data can be transmitted through the sensor nodes in minimum energy.

\section{REFERENCES}

[1] FU, Chunyao, Zhifang JIANG, and Wei WEI2and Ang WEI. "An Energy Balanced Algorithm of LEACH Protocol in WSN." International Journal of Computer Science (2013).

[2] Jing, Yang, Li Zetao, and Lin Yi. "An improved routing algorithm based on LEACH for wireless sensor networks." Control and Decision Conference (CCDC), 2013 25th Chinese. IEEE, 2013.

[3] Beiranvand, Zahra, Ahmad Patooghy, and Mahdi Fazeli. "ILEACH: An efficient routing algorithm to improve performance $\&$ to reduce energy consumption in Wireless Sensor Networks." Information and Knowledge Technology (IKT), 2013 5th Conference on. IEEE, 2013.

Xu, Jia, Ning Jin, Xizhong Lou, Ting Peng, Qian Zhou, and Yanmin Chen. "Improvement of LEACH protocol for WSN.", In Fuzzy Systems and Knowledge Discovery (FSKD), 2012 9th International Conference on, pp. 21742177. IEEE, 2012.

[5] Sipon, Miah Md, and Koo Insoo. "Performance analysis of ILEACH and LEACH protocols for wireless sensor networks." Journal of information and communication convergence engineering 10.4 (2012): 384-389.

[6] Liu, Yi, Shan Zhong, Licai You, Bu Lv, and Lin Du. "A Low Energy Uneven Cluster Protocol Design for Wireless Sensor Network." Int'1 J. of Communications, Network and System Sciences 5 (2012): 86.

[7] Haneef, Muhammad, Zhou Wenxun, and Zhongliang Deng. "MG-LEACH: Multi group based LEACH an energy efficient routing algorithm for Wireless Sensor Network." Advanced Communication Technology (ICACT), 2012 14th International Conference on. IEEE, 2012.

[8] Aslam, M., Nadeem Javaid, A. Rahim, U. Nazir, Ayesha Bibi, and Z. A. Khan. "Survey of extended LEACH-Based clustering routing protocols for wireless sensor networks."In High Performance Computing and Communication \& 2012 IEEE 9th International Conference on Embedded Software and Systems (HPCC-ICESS), 2012 IEEE 14th International Conference on, pp. 1232-1238. IEEE, 2012.

[9] Maraiya, Kiran, Kamal Kant, and Nitin Gupta. "Wireless sensor network: a review on data aggregation." International Journal of Scientific \& Engineering Research 2.4 (2011): 1-6.

[10] Raju, G. T., D. K. Ghosh, T. Satish Kumar, S. Kavyashree, and V. Nagaveni. "Wireless sensor network lifetime optimization." (2011): 244-248.

[11] Li, Yuling, Luwei Ding, and Feng Liu. "The improvement of LEACH protocol in WSN." Computer Science and Network Technology (ICCSNT), 2011 International Conference on. Vol. 2.IEEE, 2011.

[12] Jung, W. S., Lim, K. W., Ko, Y. B., \& Park, S. J. (2009, February). A hybrid approach for clustering-based data aggregation in wireless sensor networks. In Digital Society, 
2009. ICDS'09.Third International Conference on (pp. 112117). IEEE.

[13] Marta, Mirela, and Mihaela Cardei. "Using sink mobility to increase wireless sensor networks lifetime." World of Wireless, Mobile and Multimedia Networks, 2008. WoWMoM 2008.2008 International Symposium on a. IEEE, 2008.

[14] Basagni, Stefano, Alessio Carosi, Emanuel Melachrinoudis, Chiara Petrioli, and Z. Maria Wang. "Controlled sink mobility for prolonging wireless sensor networks lifetime." Wireless Networks 14, no. 6 (2008).

[15] Yiming, Fan, and Yu Jianjun."The communication protocol for wireless sensor network about LEACH." Computational Intelligence and Security Workshops, 2007.CISW 2007.International Conference on. IEEE, 2007.

[16] Mr. Santosh. Irappa. Shirol, Ashok Kumar. N, Mr. Kalmesh. M. Waderhatti, "Advanced-LEACH Protocol of Wireless Sensor network", IJETT - Volume4 Issue6- June 2013.

[17] Chunyao FU, Zhifang JIANG, Wei WEI and Ang WEI," An Energy Balanced Algorithm of LEACH Protocol in WSN", IJCSI-2013.

[18] Rajashree. V. Biradar (1), V.C .Patil (2), Dr. S. R. Sawant (3), Dr. R. R. Mudholkar, "Classification and Comparison of Routing Protocols In Wireless Sensor Networks", UbiCC Journal.

[19] Ranganathan Vidhyapriya and Ponnusamy Vanathi, "Energy Efficient Data Compression in Wireless Sensor Networks", iajit-2009.

[20] Simrandeep kaur, Student1 ; V.Sulochana Verma,” Design and Implementation of LZW Data Compression Algorithm", (IJIST) V

[21] Muhammad Nasir Khan* and Mohsin Jamil," Performance Improvement in Lifetime and Throughput of LEACH", ijst 2016.

[22] Dheemanth H N," LZW Data Compression", AJER-2014.

[23] Md. Sipon Miah and Insoo Koo," Performance Analysis of ILEACH and LEACH Protocols for Wireless Sensor Networks", jicce-2012.

[24] Shekhar Vyas1, Pinaki A. Ghosh2, " Review and Proposed Work for MODI-LEACH for Improvement of LEACH for Energy Consumption in Wireless Sensor Networks", JAERD-2014.

[25] Shahina Sheikh, Ms. Hemlata Dakhore, "Data Compression Techniques for Wireless Sensor Network", IJCSIT-2015.

[26] Shikha Gupta, Shikha Khanna, "Survey of Leach and Related Protocols under Wireless Sensor Networks", IJRREST-2015.

[27] MS. Manisha Yadav, Mrs. Moumita Ghosh," Performance analysis of different types of LEACH protocol depending on various parameters", IRJET-2016.

[28] Karthick .K et al, “An Energy-Saving Routing Algorithm for Wireless Body Sensor Network using Data Compression Technique", ijca-2015.
[29] Satyajeet R. Shinge, "Increase the Lifetime of Wireless Sensor Network using Clustering and Compression", ijca2015.

[30] S.Mohamed Saleem, "Evaluating Effectiveness of Data Transmission and Compression Technique in Wireless Sensor Networks", ijarcsse-2013.

[31] R. Swathi, "An Energy Efficient Routing Algorithm based on Data Compression in LEACH-C", ijert-2014.

[32] Mathapati, Basavaraj S., Siddarama R. Patil, and V. D. Mytri. "Energy Efficient Reliable Data Aggregation Technique for Wireless Sensor Networks."Computing Sciences (ICCS), 2012 International Conference on. IEEE, 2012.

[33] Maraiya, Kiran, Kamal Kant, and Nitin Gupta. "Wireless sensor network: a review on data aggregation." International Journal of Scientific \& Engineering Research 2.4 (2011): 1-6.

[34] Maraiya, Kiran, Kamal Kant, and Nitin Gupta. "Efficient cluster head selection scheme for data aggregation in wireless sensor network." International Journal of Computer Applications 23.9 (2011): 10-18.

[35] Li, Hongjuan, Kai Lin, and Keqiu Li. "Energy-efficient and high-accuracy secure data aggregation in wireless sensor networks." Computer Communications 34.4 (2011): 591597.

[36] Zanjani, Motahareh Bahrami, Reza Monsefi, and Arash Boustani. "Energy efficient/highly secure data aggregation method using tree-structured orthogonal codes for Wireless Sensor Networks." Software Technology and Engineering (ICSTE), 2010 2nd International Conference on. Vol. 2.IEEE, 2010.

[37] Bista, Rabindra, Kim Hee-Dae, and Jae-Woo Chang. "A new private data aggregation scheme for wireless sensor networks." Computer and Information Technology (CIT), 2010 IEEE 10th International Conference on. IEEE, 2010.

[38] Xiao, Haitao, Tansheng Li, Harutoshi Ogai, Xiaohong Zou, Takenari Otawa, Shinya Umeda, and Takunori Tsuji. "The health monitoring system based on distributed data aggregation for WSN used in bridge diagnosis." In SICE Annual Conference 2010, Proceedings of, pp. 2134-2138. IEEE, 2010.

[39] Yu, Bo, Jianzhong Li, and Yingshu Li. "Distributed data aggregation scheduling in wireless sensor networks." INFOCOM 2009, IEEE. IEEE, 2009.

[40] Jung, W. S., Lim, K. W., Ko, Y. B., \& Park, S. J. (2009, February). A hybrid approach for clustering-based data aggregation in wireless sensor networks. In Digital Society, 2009. ICDS'09. Third International Conference on (pp. 112117). IEEE.

[41] Ozdemir, Suat. "Functional reputation based reliable data aggregation and transmission for wireless sensor networks." Computer Communications 31.17 (2008): 3941-3953.

[42] Liao, Wen-Hwa, Yucheng Kao, and Chien-Ming Fan. "An ant colony algorithm for data aggregation in wireless sensor networks." Sensor Technologies and Applications, 
2007.SensorComm 2007. International Conference on IEEE, 2007.

[43] S. Manfredi, "Reliable and Energy-Efficient Cooperative Routing Algorithm For Wireless Monitoring Systems," IET Wireless Sensor Systems, vol. 2, no. 2, pp. 128-135, 2012.

[44] T. Y. Tsai, W. C. Lan, C. Liu, and M. T. Sun, "Distributed Compressive Data Aggregation in Large-Scale Wireless Sensor Networks," Journal of Advances in Computer Networks, vol. 1, no. 4, pp. 295-300, 2012.

[45] D. C. Hoang, R. Kumar, and S. K. Panda, "Optimal Data Aggregation Tree in Wireless Sensor Networks Based on Intelligent Water Drops Algorithm, IET Wireless Sensor Systems, vol. 2, no. 3, pp. 282-292, 2012.

[46] C. M. Yu and J. H. Lin, "Enhanced Bluetree: a mesh topology approach forming Bluetooth Scatternet," IET Wireless Sensor Systems, vol. 2, no. 4, pp. 409-415, 2012.
[47] R. M. Islam, Y. A. Abdulrahman, T. A. Rahman, “An Improved ITU-R rain Attenuation Prediction Model Over Terrestrial Microwave Links in Tropical Region," EURASIP Journal on Wireless Communication and Networking, vol. 189, pp. 1-9, 2012.

[48] P. L. Shrestha, M. Hempel, H. Sharif, and H. Chen, "Modeling Latency and Reliability of Hybrid technology Networking," IEEE Sensor Journal, vol. 13, no. 10, pp. 3616-3624, 2012.

[49] A. E. A. A. Abdulla, H. Nishiyama, and N. Kato, "Extending the Lifetime of Wireless Sensor Networks: A Hybrid Routing Algorithm, Elsevier, vol. 35, no. 9, pp. 1056-1063, 2012.

[50] A. Jain and N. Hemrajani, "Performance Analysis \& Evaluation by Simulation of Wireless Sensor Network Using Energy Efficient Algorithm," International Journal of Application or Innovation in Engineering \& Management, vol. 2, no. 1, pp. 236-239, 2013

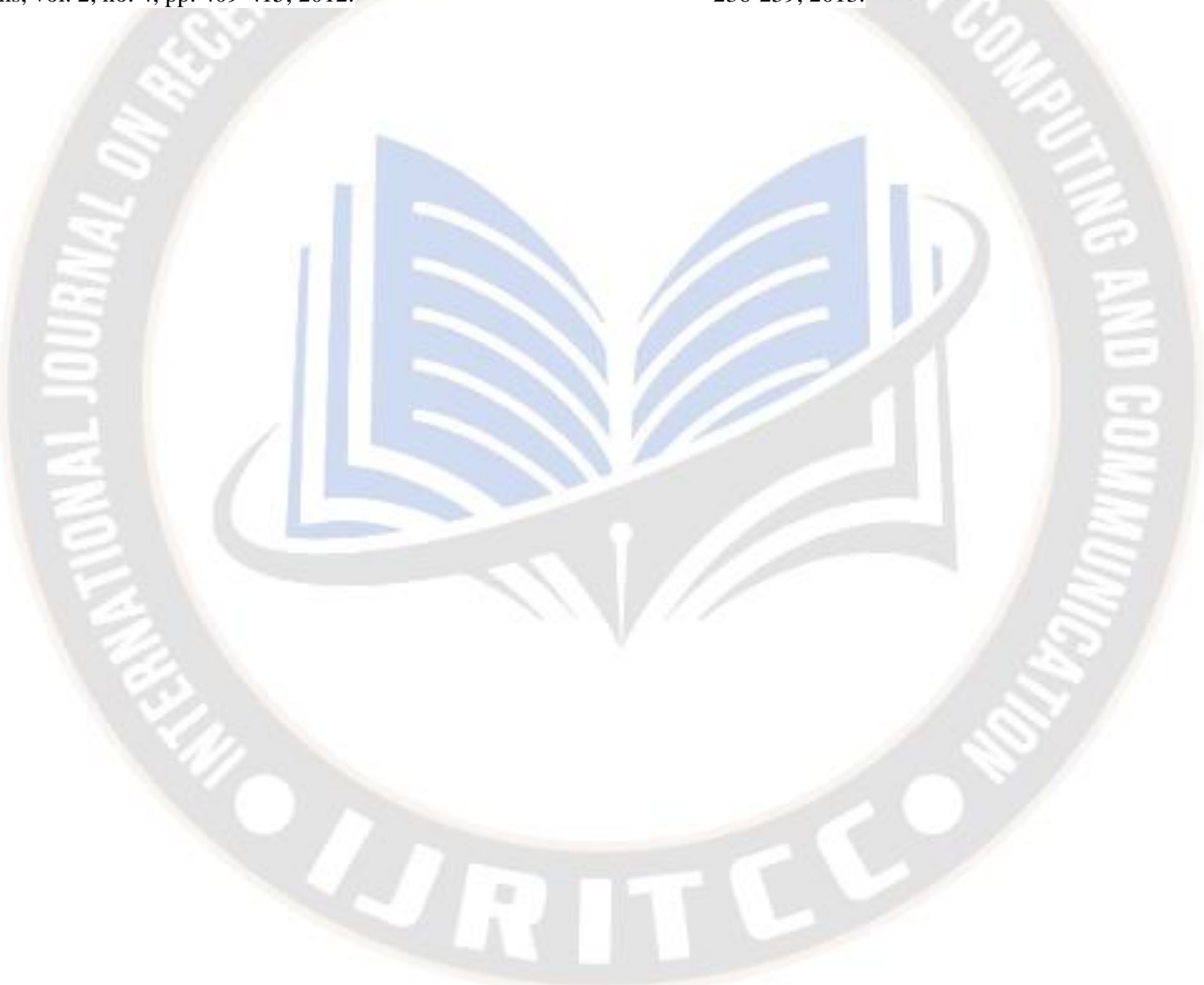

